\title{
Is the time lag-luminosity relation of gamma-ray bursts a consequence of the Amati relation?
}

\author{
M. Hafizi ${ }^{1}$ and R. Mochkovitch ${ }^{2}$ \\ 1 Tirana University, Faculty of Natural Sciences, Tirana, Albania \\ 2 Institut d'Astrophysique de Paris - UMR 7095 CNRS et Université Pierre et Marie Curie, 98 bis, boulevard Arago, 75014 Paris, \\ France \\ e-mail: mochko@iap.fr
}

Received 7 July 2005 / Accepted 24 October 2006

\begin{abstract}
Context. The lag-luminosity relation (LLR) provides a way of estimating GRB luminosity by measuring the spectral lags between different energy bands.

Aims. We want to understand the origin of the LLR and test its validity. This appears especially important if the LLR is to be used as a distance indicator.

Methods. We perform a linear analysis of the lag between two spectral bands. The lag is obtained as the time interval between the maxima of a given pulse in the two bands.

Results. We get a simple expression for the lag, which shows in a very simple way how it is related to the spectral evolution of the burst via the variation of the peak energy and spectral indices. When this expression is coupled to the Amati relation, it leads to a LLR that agrees with the observational results only if the burst's spectral evolution is limited to a decrease in peak energy during pulse decay. However, when the variation of the spectral indices is also taken into account, the predicted LLR differs from the observed one. Conclusions. We briefly discuss some ways to solve this problem, such as a possible correlation between pulse spikiness and burst luminosity.
\end{abstract}

Key words. gamma rays: bursts - radiation mechanisms: non thermal

\section{Introduction}

The problem of the distance to gamma-ray bursts remained unsolved until the discovery of the afterglows by Beppo-SAX. Redshifts are now obtained from optical spectra of the afterglow itself or of the host galaxy when the afterglow has faded away. Using the known redshifts, it became possible to calibrate relations by linking absolute burst outputs (luminosity or total radiated energy) and quantities directly available from the observations in gamma-rays. A Cepheid-like relation between variability and luminosity was proposed, for example, by Reichart et al. (2001). More recently, Atteia (2003) used the Amati relation (Amati et al. 2002) to introduce "pseudo-redshifts" which could be useful to rapidly identify high- $z$ GRBs from their gamma-ray properties alone. In this paper we concentrate on the time lag-luminosity relation (LLR) discovered by Norris et al. (2000). The lags were computed by Norris et al. using the burst profiles in BATSE band $1(20-50 \mathrm{keV})$ and $3(100-300 \mathrm{keV})$. They find that the time lag $\Delta t_{13}$ anticorrelates with burst luminosity and propose the following power law relation

$L=1.3 \times 10^{53}\left(\Delta t_{13} / 0.01 \mathrm{~s}\right)^{-1.15} \mathrm{erg} \mathrm{s}^{-1}$.

The origin of the LLR was then investigated by Kocevski \& Liang (2003), Ryde (2005), and Ryde et al. (2005), who found that the observed lags are a consequence of the burst's spectral evolution. In this contribution we perform a new analysis of the origin of lags and discuss how the LLR may be linked to the Amati relation and possibly also to the variability-luminosity relation.

\section{Count rates in different energy bands}

We consider a spectral band $\left[E_{i}, E_{j}\right]$ and assume a spectrum shape consisting of two smoothly-connected power laws of respective slopes $\alpha$ and $\beta$ at low and high energy (Band et al. 1993). The count rate $N_{i j}(t)$ in band $\left[E_{i}, E_{j}\right]$ reads

$N_{i j}(t)=A(t) \int_{x_{i}}^{x_{j}} \mathcal{B}_{\alpha \beta}(x) \mathrm{d} x$

where the function $A(t)$ depends on time alone. The limits of the integral are $x_{i, j}=E_{i, j}(1+z) / E_{\mathrm{p}}(t), z$ being the redshift of the source, $E_{\mathrm{p}}(t)$ the peak energy of the instantaneous spectrum (in source rest frame) and $\mathcal{B}_{\alpha \beta}(x)$ the spectrum shape.

Considering now another spectral band $\left[E_{k}, E_{l}\right]$, we can relate $N_{k l}(t)$ to $N_{i j}(t)$ in the following way

$$
\begin{aligned}
N_{k l}(t) & =N_{i j}(t) \times \frac{\int_{x_{k}}^{x_{l}} \mathcal{B}_{\alpha \beta}(x) \mathrm{d} x}{\int_{x_{i}}^{x_{j}} \mathcal{B}_{\alpha \beta}(x) \mathrm{d} x} \\
& =N_{i j}(t) \times \mathcal{F}_{i j k l}\left[E_{\mathrm{p}}(t), \alpha(t), \beta(t)\right]
\end{aligned}
$$

where $\mathcal{F}_{i j k l}$ can be seen as the "spectral correction" between bands $\left[E_{i}, E_{j}\right]$ and $\left[E_{k}, E_{l}\right]$. We simplify the notation by considering only BATSE bands $1[20,50 \mathrm{keV}]$ and $3[100,300 \mathrm{keV}]$ so that we have

$N_{3}(t)=N_{1}(t) \times \mathcal{F}_{13}\left[E_{\mathrm{p}}, \alpha, \beta\right]$ 
with

$\mathcal{F}_{13}=\frac{\int_{100(1+z) / E_{\mathrm{p}}}^{300(1+z) / E_{\mathrm{p}}} \mathcal{B}_{\alpha \beta}(x) \mathrm{d} x}{\int_{20(1+z) / E_{\mathrm{p}}}^{50(1+z) / E_{\mathrm{p}}} \mathcal{B}_{\alpha \beta}(x) \mathrm{d} x}$.

We then assume that a given pulse in the burst profile reaches its maximum at a time $t_{1}$ (resp. $t_{3}$ ) in band 1 (resp. 3) and we estimate the lag by the difference

$\Delta t_{13}=t_{1}-t_{3}$

Since in most cases the observed lags are small compared to the pulse duration, we evaluate $\Delta t_{13}$ from a linear analysis of the pulse shape around $t_{1}$. Using $\left.\frac{\mathrm{d} N_{1}}{\mathrm{~d} t}\right|_{t=t_{1}}=\dot{N}_{1}\left(t_{1}\right)=0$, we can write

$N_{1}(t) \simeq N_{1}\left(t_{1}\right)+\frac{1}{2} \ddot{N}_{1}\left(t_{1}\right)\left(t-t_{1}\right)^{2}$,

while the spectral correction gives to same order

$\mathcal{F}_{13}(t)=\mathcal{F}_{13}\left(t_{1}\right)+\dot{\mathcal{F}}_{13}\left(t_{1}\right)\left(t-t_{1}\right)+\frac{1}{2} \ddot{\mathcal{F}}_{13}\left(t_{1}\right)\left(t-t_{1}\right)^{2}$

with $\dot{\mathcal{F}}_{13}\left(t_{1}\right)$ and $\ddot{\mathcal{F}}_{13}\left(t_{1}\right)$ being related to the partial derivatives of $\mathcal{F}_{13}$ with respect to $E_{\mathrm{p}}, \alpha$, and $\beta$. For $\dot{\mathcal{F}}_{13}\left(t_{1}\right)$ we have

$\dot{\mathcal{F}}_{13}\left(t_{1}\right)=\left.\frac{\partial \mathcal{F}_{13}}{\partial E_{\mathrm{p}}}\right|_{t_{1}} \dot{E}_{\mathrm{p}}\left(t_{1}\right)+\left.\frac{\partial \mathcal{F}_{13}}{\partial \alpha}\right|_{t_{1}} \dot{\alpha}\left(t_{1}\right)+\left.\frac{\partial \mathcal{F}_{13}}{\partial \beta}\right|_{t_{1}} \dot{\beta}\left(t_{1}\right)$,

while $\ddot{\mathcal{F}}_{13}\left(t_{1}\right)$ contains nine terms. We now compute the logarithmic derivative of $N_{3}(t)$ to the first order in $\left(t-t_{1}\right)$

$$
\begin{aligned}
\frac{\dot{N}_{3}(t)}{N_{3}(t)}= & \frac{\dot{N}_{1}(t)}{N_{1}(t)}+\frac{\dot{\mathcal{F}}_{13}(t)}{\mathcal{F}_{13}(t)}=\frac{\ddot{N}_{1}\left(t_{1}\right)}{N_{1}\left(t_{1}\right)}\left(t-t_{1}\right) \\
& +\frac{\dot{\mathcal{F}}_{13}\left(t_{1}\right)}{\mathcal{F}_{13}\left(t_{1}\right)}+\left[\frac{\ddot{\mathcal{F}}_{13}\left(t_{1}\right)}{\mathcal{F}_{13}\left(t_{1}\right)}-\left(\frac{\dot{\mathcal{F}}_{13}\left(t_{1}\right)}{\mathcal{F}_{13}\left(t_{1}\right)}\right)^{2}\right]\left(t-t_{1}\right) .
\end{aligned}
$$

The bracket contains many terms involving partial derivatives of $\mathcal{F}_{13}$ to the first and second order, but it turns out that they are essentially negligible for the final numerical results. Solving Eq. (10) to get $t_{3}$, such as $\dot{N}_{3}\left(t_{3}\right)=0$, finally yields

$\frac{\Delta t_{13}}{t_{\mathrm{p}}} \simeq \frac{f_{13, E} \dot{e}_{\mathrm{p}}+f_{13, \alpha} \dot{a}+f_{13, \beta} \dot{b}}{C_{1}}$

with

$$
\begin{aligned}
f_{13, X} & =\left.\frac{\partial \log \mathcal{F}_{13}}{\partial \log X}\right|_{t_{1}}, \quad \dot{e}_{\mathrm{p}}=\frac{\dot{E}_{\mathrm{p}}}{E_{\mathrm{p}}} t_{\mathrm{p}} \\
\dot{a} & =\frac{\dot{\alpha}}{\alpha} t_{\mathrm{p}}, \quad \dot{b}=\frac{\dot{\beta}}{\beta} t_{\mathrm{p}} \text { and } \frac{C_{1}}{t_{\mathrm{p}}^{2}}=\frac{\ddot{N}_{1}\left(t_{1}\right)}{N_{1}\left(t_{1}\right)}
\end{aligned}
$$

where $t_{\mathrm{p}}$ is the characteristic duration of the pulse. For two given spectral bands and an assumed spectral shape, Eq. (11) provides a linear estimate of the lag, which directly shows how it is related to burst spectral evolution via the temporal derivatives of $E_{\mathrm{p}}$, $\alpha$ and $\beta$. The "curvature parameter" $\left|C_{1}\right|$ depends on the pulse shape at maximum, large (resp. small) $\left|C_{1}\right|$ values corresponding to spiky (resp. broad) pulses.

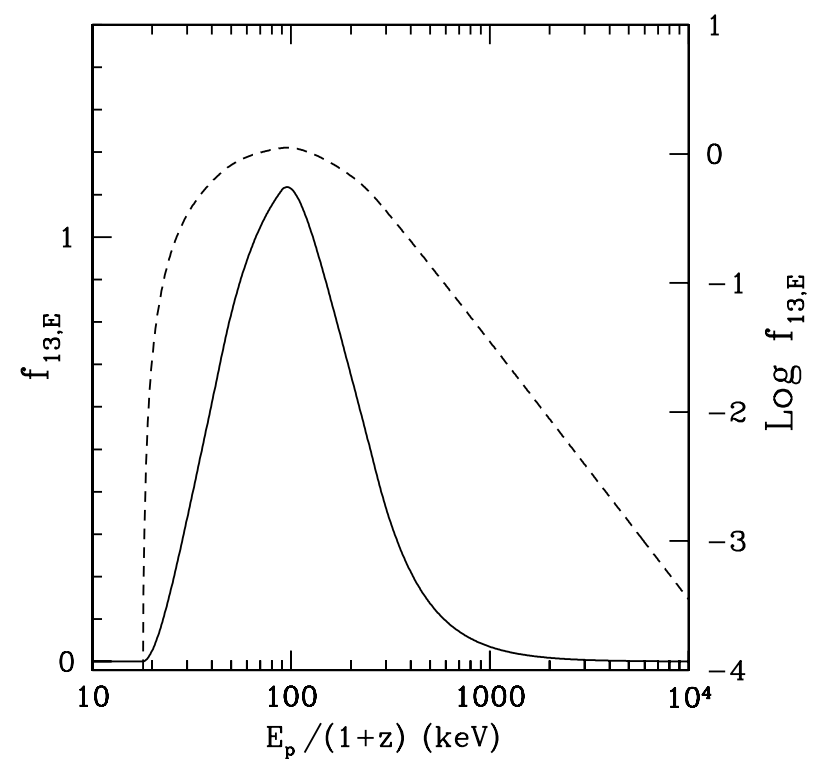

Fig. 1. The function $f_{13, E}$ given by Eq. (14) plotted as a function of the observed peak energy for $\alpha=-1$ and $\beta=-2.25$; full line: linear scale; dashed line: logarithmic scale.

\section{The lag luminosity relation}

Equation (11) gives the lag between BATSE bands 1 and 3 if the values of $E_{\mathrm{p}}, \alpha, \beta$, their time derivatives, and the pulse shape are known at maximum. It will become a LLR if these parameters can be related in some way to the luminosity. The Amati relation (Amati et al. 2002) provides such a link but, in its most studied version, it connects the isotropic energy in gamma-rays to the $E_{\mathrm{p}}$ value of the global spectrum. However, it has been suggested that a similar relation may exist between $E_{\mathrm{p}}$ and the luminosity. Yonetoku et al. (2004) find, for example, a relation between the maximum luminosity and the global $E_{\mathrm{p}}$, while Ghirlanda et al. (2005) propose a relation between the values of $E_{\mathrm{p}}$ and the luminosity both taken at pulse maximum

$E_{\mathrm{p}}=380\left(\frac{L}{1.6 \times 10^{52} \mathrm{erg} \mathrm{s}^{-1}}\right)^{0.43} \mathrm{keV}$.

If a substantial fraction of bursts satisfy Eq. (13), it will, together with Eq. (11), lead to a LLR that can be compared to the observational data.

\subsection{A (too) simple example}

In this section we limit the burst's spectral evolution to the variation of $E_{\mathrm{p}}$ only and write $\dot{a}=\dot{b}=0$. Equation (11) then simply becomes

$$
\frac{\Delta t_{13}}{t_{\mathrm{p}}} \simeq \frac{f_{13, E} \dot{e}_{\mathrm{p}}}{C_{1}} \quad \text { with } \quad f_{13, E}=\left.\frac{\partial \log \mathcal{F}_{13}}{\partial \log E_{\mathrm{p}}}\right|_{t_{1}, \alpha, \beta} \text {. }
$$

The function $f_{13, E}$ tends towards 0 for the highest and lowest values of $E_{\mathrm{p}}$, while it is at its maximum for $50 \lesssim E_{\mathrm{p}} /(1+z) \lesssim$ $100 \mathrm{keV}$. This is illustrated in Fig. 1 and can be easily understood, since for $E \ll E_{\mathrm{p}}$ (resp. $E \gg E_{\mathrm{p}}$ ) the Band function $\mathcal{B}(x)$ behaves as $x^{\alpha}$ (resp. $\left.x^{\beta}\right)$. Therefore for high $E_{\mathrm{p}}$ values $\left(E_{\mathrm{p}} /(1+z) \gg 300 \mathrm{keV}\right)$

$\mathcal{F}_{13} \simeq \frac{300^{1+\alpha}-100^{1+\alpha}}{50^{1+\alpha}-20^{1+\alpha}}=C^{\mathrm{st}}$ and $f_{13, E}=0$ 


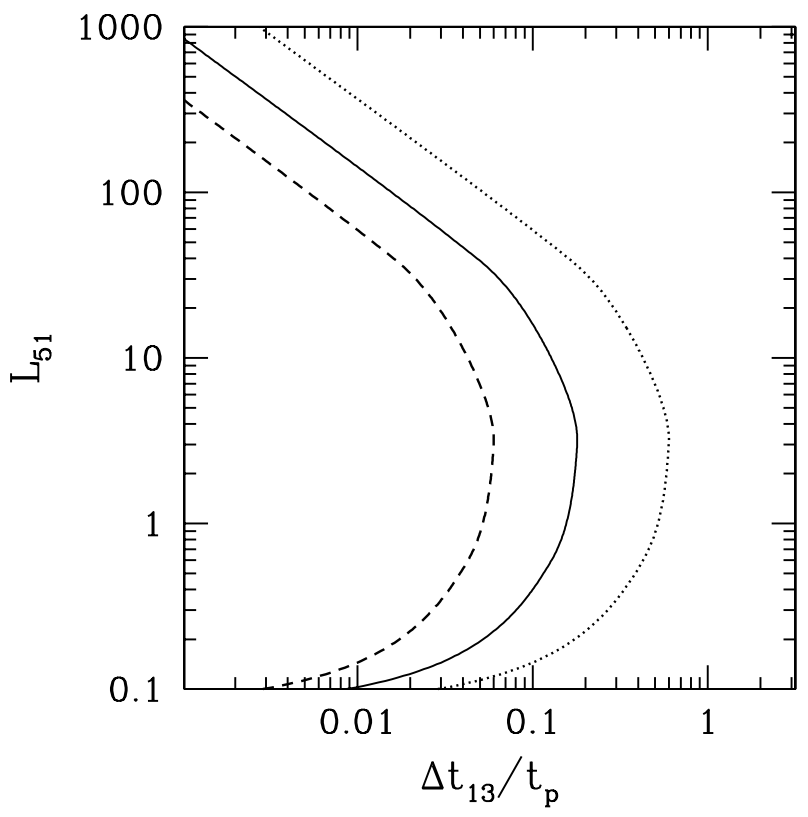

Fig. 2. Lag-luminosity relation resulting from Eq. (14) which limits the spectral evolution to a decrease of $E_{\mathrm{p}}$ during pulse decay ( $\alpha$ and $\beta$ being kept constant respectively equal to -1 and -2.25$)$. The three lines cover an order of magnitude in $\left|\dot{e}_{\mathrm{p}} / C_{1}\right|$ from 0.1 (dashed line) to 0.3 (full line) and 1 (dotted line). A redshift value $z=1$ has been assumed.

a similar result with $\beta$ replacing $\alpha$ being obtained for low $E_{\mathrm{p}} /(1+$ $z) \ll 20 \mathrm{keV}$. At intermediate values, $50 \lesssim E_{\mathrm{p}} /(1+z) \lesssim 100 \mathrm{keV}$, and making the rough approximation that $\mathcal{B}(x) \propto x^{\alpha}$ in band 1 while $\mathcal{B}(x) \propto x^{\beta}$ in band 3, we obtain

$\mathcal{F}_{13} \simeq \frac{300^{1+\beta}-100^{1+\beta}}{50^{1+\alpha}-20^{1+\alpha}}\left(\frac{E_{\mathrm{p}}}{1+z}\right)^{\alpha-\beta}$

and therefore

$f_{13, E}=\alpha-\beta$.

Finally it can be shown that the shape of $\mathcal{B}(x)$ at small $x, \mathcal{B}(x)=$ $x^{\alpha}[1-(2+\alpha) x]$ leads to a power law behavior for $f_{13, E}$ at large $E_{\mathrm{p}}$ as seen in Fig. 1.

If the Amati-like relation (Eq. (13)) is satisfied, high luminosity bursts will have a large $E_{\mathrm{p}}$ and therefore a small lag, while lags will be comparatively large for bursts with $E_{\mathrm{p}}$ in the range $50(1+z)-100(1+z) \mathrm{keV}$, i.e. $L \sim 4 \times 10^{50}(1+z)^{2.3} \mathrm{erg} \mathrm{s}^{-1}$ at pulse maximum. Equation (14) also predicts that, for a given luminosity and spectral evolution, spiky bursts (large $\left|C_{1}\right|$ ) will have smaller lags than bursts with broad pulses (small $\left|C_{1}\right|$ ), in agreement with observations (Hakkila \& Giblin 2006). The LLR obtained with this simple model is represented in Fig. 2 for different values of the ratio $\left|\dot{e}_{\mathrm{p}} / C_{1}\right|$ and a typical burst redshift $z=1$. A large (resp. small) ratio corresponds to a faster (resp. slower) spectral evolution or to a broader (resp. spikier) pulse. At high luminosity $\left(L>10^{52} \mathrm{erg} \mathrm{s}^{-1}\right)$, the LLR has a power law behavior since

$\frac{\mathrm{d} \log L}{\mathrm{~d} \log \Delta t}=\left(\frac{\mathrm{d} \log L}{\mathrm{~d} \log E_{\mathrm{p}}}\right)\left(\frac{\mathrm{d} \log E_{\mathrm{p}}}{\mathrm{d} \log \Delta t}\right)$

the first factor being given by the Amati relation (Eq. (13)), while the second results from the spectrum shape. From Eq. (14) we have

$\frac{\mathrm{d} \log E_{\mathrm{p}}}{\mathrm{d} \log \Delta t}=\frac{\mathrm{d} \log E_{\mathrm{p}}}{\mathrm{d} \log f_{13, E}}$

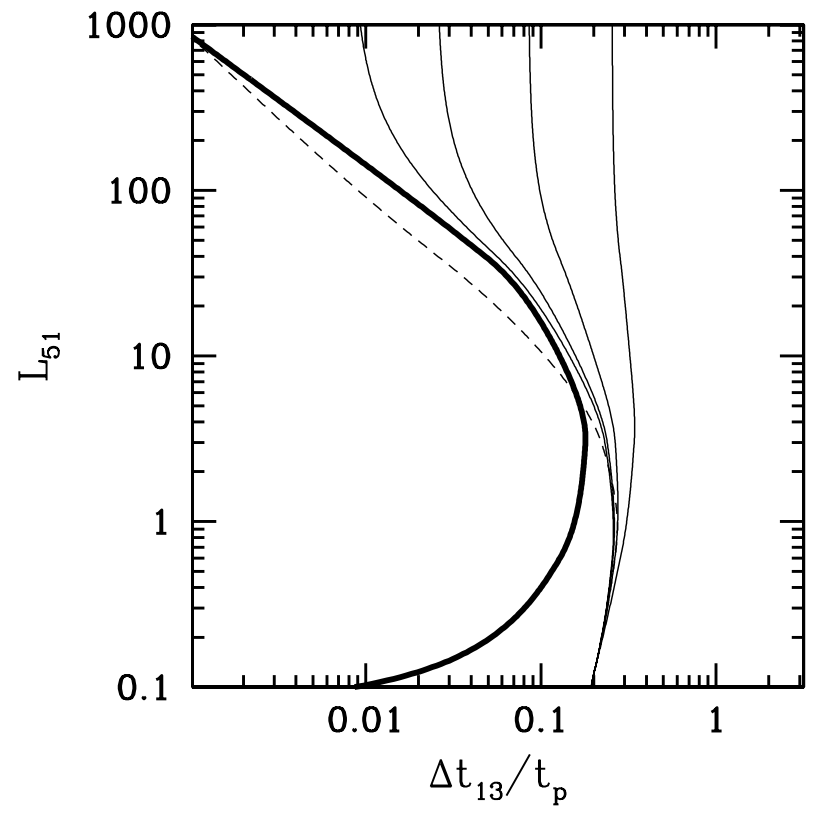

Fig. 3. LLR with $\left|\dot{e}_{\mathrm{p}} / C_{1}\right|=0.3$, but now also including the variation in the low and high-energy spectral indices. The thin lines correspond respectively (from left to right) to $\dot{a}=0.01,0.03,0.1$, and 0.3 (and have all $\dot{b}=0.1$ ), while the thick full line represents the $\dot{a}=\dot{b}=0$ case. The dashed line is obtained with $\dot{a}=\dot{b}=0.1$ and a varying curvature parameter given by Eq. (20).

which is a constant at high $E_{\mathrm{p}}$ (and hence luminosity) values (see Fig. 1). However at luminosities smaller than $10^{52} \mathrm{erg} \mathrm{s}^{-1}$ the model predicts that the power law behavior of the LLR should break down with the lag passing through a maximum and then decreasing. This clearly contradicts GRB 980425, which has both a very low luminosity and a large lag. But GRB 980425 does not satisfy the Amati relation so that its departure from the LLR is not surprising. One should instead consider that this burst has a broad temporal profile, i.e. a small $\left|C_{1}\right|$ and an $E_{\mathrm{p}}$ of $138 \mathrm{keV}$ (Ghisellini et al. 2006) corresponding to the maximum of $f_{13, E}$ (see Fig. 1) and therefore to a large expected lag.

\subsection{A more complete study}

The spectral evolution of GRBs is, however, not limited to a decrease in $E_{\mathrm{p}}$ during pulse decay. A hard-to-soft evolution is also observed for the spectral indices $\alpha$ and $\beta$. In some extreme cases $\alpha$ has been seen to decrease from $\alpha \sim 1.5$ (a value a priori excluded by the synchrotron model) to about -0.5 in just a few seconds (Crider et al. 1997). When the variation in the spectral indices is included in our linear analysis, it no longer predicts a vanishing lag for high or low $E_{\mathrm{p}}$ and $L$ values since now $f_{13, \alpha} \neq 0$ when $E_{\mathrm{p}} \rightarrow \infty$ and $f_{13, \beta} \neq 0$ when $E_{\mathrm{p}} \rightarrow 0$ (for example $\lim _{E_{\mathrm{p}} \rightarrow \infty} f_{13, \alpha} \simeq-1.7$ for $\alpha=-1$ and $\beta=-2.25$ ). The lag then reaches a constant limiting value at low and high luminosities, where it apparently contradicts the observed LLR. This is shown in Fig. 3 where our calculated LLR has been plotted for different values of $\dot{a}$ and a fixed $\dot{b}=0.1$. Even a moderate variation in the spectral indices has a dramatic effect on the LLR and the global agreement with the Norris et al. (2000) results that was found in the last section is now lost. 


\section{Discussion}

These results clearly disagree with the observational data for the most luminous GRBs. Therefore if real bursts do satisfy the LLR proposed by Norris et al. (2000), a solution has to be found for the apparent discrepancy between our analysis and the observations:

(i) A first option could be that in most cases the variation in the spectral index $\alpha$ is small, at least around pulse maximum. However this does not seem to be the case for the bright events for which a detailed, time resolved, spectral analysis has been possible (Preece et al. 2000). Moreover, the constraint on any variation in $\alpha$ appears so severe (only the LLR with $\dot{a}=0.01$ in Fig. 3 is marginally compatible with the Norris et al. results) that it seems difficult to expect it will be satisfied by a large fraction of GRBs.

(ii) A more interesting possibility would be that a relation may exist between the curvature parameter $\left|C_{1}\right|$ and the luminosity, bursts with spiky pulses being more luminous than bursts with broad pulses. This might be a different way to express the variability-luminosity relation proposed by Reichart et al. (2001). We tried, for example, a simple linear expression of the form

$\left|C_{1}\right|=2+0.2\left(L_{51}-1\right)$

where the resulting LLR with $\left|\dot{e}_{\mathrm{p}} / C_{1}\right|=0.3$ and $\dot{a}=\dot{b}=0.1$ is represented in Fig. 3. In spite of the variation in $\alpha$, it now gives again very small lags at high luminosity because the pulses are then much spikier than at low luminosity.

\section{Conclusion}

We have performed a linear analysis of the time lag between two spectral bands and have obtained a simple relation (Eq. (11)) which clarifies how the lag is related to the burst spectral evolution. When this relation is used in conjunction with the Amati relation it leads to a satisfactory LLR only if the spectral evolution of GRBs is limited to a decrease of $E_{\mathrm{p}}$ during pulse decay. If the variation in the spectral indices is also included, the lag does not decrease any longer to low values, even at very high burst luminosity. We have briefly discussed the possibility that short lags might be recovered if burst luminosity is correlated to the shape of the pulses, bursts with spiky pulses being more luminous than bursts with broad pulses. The available sample of GRBs with both measured lags and known distance is still small but should increase with SWIFT. This will allow constraining tests of the results presented in this paper.

Acknowledgements. The authors thank Frédéric Daigne for helpful discussions and Felix Ryde for communicating several of his results prior to publication. They also thank the anonymous referee whose comments have considerably improved an initial version of this paper.

\section{References}

Amati, L., Frontera, F., Tavani, M., et al. 2002, A\&A, 390, 81 Atteia, J. L. 2003, A\&A, 407, L1

Band, D., Matteson, J., Ford, L., et al. 1993, ApJ, 413, 281

Crider, A., Liang, E. P., Smith, I. A., et al. 1997, ApJ, 479, L39

Ghirlanda, G., Ghisellini, G., \& Firmani, C., et al. 2005, MNRAS, 360, L45

Ghisellini, G., Ghirlanda, G., Mereghetti, S., et al. 2006 [arXiv: astro-ph/0605431]

Hakkila, J., \& Giblin, T. W. 2006 [arXiv:astro-ph/0604151]

Kocevski, D., \& Liang, E. 2003, ApJ, 594, 385

Norris, J., Marani, G., \& Bonnell, J. 2000, ApJ, 534, 248

Preece, R. D., Briggs, M. S., Mallozi, R. S., et al. 2000, ApJS, 126, 19

Reichart, D., Lamb, D., Fenimore, E., et al. 2001, ApJ, 552, 57

Ryde, F. 2005, A\&A, 429, 869

Ryde, F., Kocevski, D., Bagoly, Z., et al. 2005, A\&A, 432, 105

Yonetoku, R., et al. 2004, ApJ, 609, 935 